\title{
Global control and fast solid-state donor electron spin quantum computing
}

\author{
C. D. Hill, ${ }^{1, *}$ L. C. L. Hollenberg, ${ }^{2}$ A. G. Fowler, ${ }^{2}$ C. J. Wellard, ${ }^{2}$ A. D. Greentree, ${ }^{2}$ and H.-S. Goan ${ }^{3}$ \\ ${ }^{1}$ Centre for Quantum Computer Technology, and Department of Physics, The University of Queensland, St Lucia, QLD 4072, Australia \\ ${ }^{2}$ Center for Quantum Computer Technology, School of Physics, The University of Melbourne, Victoria 3010, Australia \\ ${ }^{3}$ Department of Physics, National Taiwan University, Taipei 106, Taiwan, Republic of China
}

(Received 24 February 2005; published 22 July 2005)

\begin{abstract}
We propose a scheme for quantum information processing based on donor electron spins in semiconductors, with an architecture complementary to the original Kane proposal. We show that a naïve implementation of electron spin qubits provides only modest improvement over the Kane scheme, however through the introduction of global gate control we are able to take full advantage of the fast electron evolution timescales. We estimate that the latent clock speed is 100-1000 times that of the nuclear spin quantum computer with the ratio $T_{2} / T_{\text {ops }}$ approaching the $10^{6}$ level.
\end{abstract}

DOI: $10.1103 / P h y s R e v B .72 .045350$

PACS number(s): 03.67.Lx

\section{INTRODUCTION}

The interest in constructing the components of a solidstate quantum computer (QC) device where the logical qubits are encoded by single donor $\operatorname{spin}^{1,2}$ or charge ${ }^{3}$ degrees of freedom is largely based on the nexus to scalable fabrication technology in the semiconductor industry. The nuclear spin Kane QC, ${ }^{1}$ is of particular interest due to the relatively long coherence timescale of P-donor nuclear spins, which bodes well for qubit storage. On the other hand, simulations of electron exchange mediated two-qubit logic gates in the Kane scheme $e^{4-6}$ showed that the gate fidelity is limited primarily by the electron coherence where the dephasing timescale was expected to be closer to the typical gate operation time of $\mathrm{O}(\mu \mathrm{s})$. Recent measurements ${ }^{7}$ indicate that the coherence time for phosphorus donor electron spins in silicon is considerably longer-greater than $60 \mathrm{~ms}$ at $T=4 \mathrm{~K}$. This surprisingly long coherence time means that donor electronspin based quantum computers may be a more desirable goal in terms of relative simplicity of qubit identification, readout, and inherent gate speed.

Proposals for donor-electron spin quantum computing as variations on the original Kane theme already exist. That of Vrijen et $a l^{2}$ based on $g$-factor engineering calls for the fabrication of complex heterostructures, and the ability to drag the electron wave function into high- $g$ regions without ionization. The "digital" quantum computer concept ${ }^{8-10}$ relies on the ability to coherently transport electron spins along the $\mathrm{Si}$-oxide interface using surface gates. The use of electron spins in quantum dot systems has been considered several times previously, for example, in GaAs systems ${ }^{11}$ and $\mathrm{Si}-\mathrm{Ge}$ heterostructures. ${ }^{12}$ A phosphorous donor electron QC based on the dipole interaction was proposed in Ref. 13. A recent review of silicon quantum-computer architectures can be found in Ref. 14.

Between the original Kane proposal and these two variants we present a new proposal for a solid-state quantum computer where the qubits are also encoded on the spins of $\mathrm{Si}: \mathrm{P}$ donor electrons, yet retaining the relative simplicity of the original Kane design. In this proposal we literally turn the Kane donor based nuclear spin QC concept inside out and couple it with new ideas for spin readout. The phosphorus donors now serve to localize the electron spins in space, and to provide local qubit addressability through the electron-nuclear hyperfine interaction. Contrary to the essential and rather complex role played by the nonlogical spins in the Kane proposal-the electron spins-here the nuclear spins are essentially frozen spectators. The donor electron spin based quantum computer has potentially an inherently faster clock speed than the nuclear spin version due to the much larger magnetic moment. To fully access this is nontrivial. By introducing new concepts in global control of spin qubits and correction of spectator evolution, we show by direct simulation that the inherent speed of the electron spin timescales can be fully exploited. Single gate operations are achieved with gate times down to tens of nanoseconds, commensurate with the exchange based CNOT gate on the order of $150 \mathrm{~ns}$. We estimate that the electron spin donor QC will have an inherent clock speed around 100 times that of the nuclear spin QC, with $T_{2} / T_{\text {ops }}$ approaching $10^{6}$ (see Table I). A summary of recent work on single donor electron spin readout ${ }^{15-19}$ completes the proposal.

This paper is organized as follows. We introduce the notion of effective single-spin gate operation through global control supplemented by only weak local control, and correction of spin spectators in the nanosecond temporal arena of fast electron spin dynamics. We contrast the gate speeds achieved with the relatively slow canonical single-spin/

TABLE I. Table summarizing the relative timescales for locally controlled nuclear (Ref. 5) and electron spin qubits compared to the globally controlled electron spin case. For both nuclear and electron spin qubits the effective dephasing time is taken to be the faster of the two, $T_{2}>60 \mathrm{~ms}$ (Ref. 7).

\begin{tabular}{ccccc}
\hline \hline Qubit & $T_{X}$ & $T_{2} / T_{X}$ & $T_{\mathrm{CNOT}}$ & $T_{2} / T_{\mathrm{CNOT}}$ \\
\hline$n$-spin & $6 \mu \mathrm{s}$ & $10^{4}$ & $16 \mu \mathrm{s}$ & $4 \times 10^{4}$ \\
$\begin{array}{c}e \text {-spin } \\
\text { (local control) } \\
e \text {-spin }\end{array}$ & $2 \mu \mathrm{s}$ & $3 \times 10^{4}$ & $\mathrm{O}(10 \mu \mathrm{s})$ & $\mathrm{O}\left(10^{3}\right)$ \\
$($ global control) & $30 \mathrm{~ns}$ & $2 \times 10^{6}$ & $148 \mathrm{~ns}$ & $6 \times 10^{5}$ \\
\hline \hline
\end{tabular}




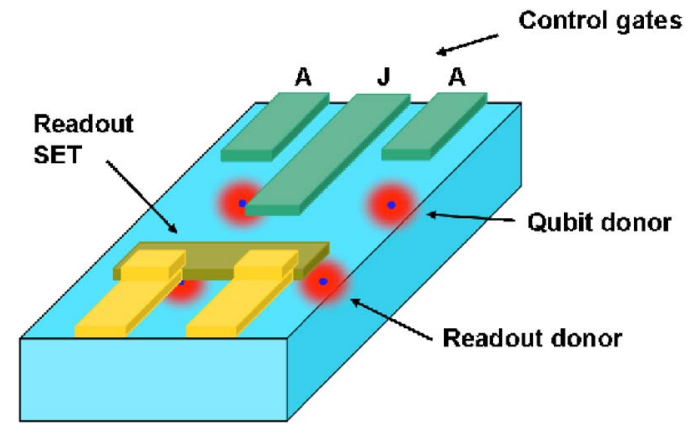

FIG. 1. (Color online) Donor electron spin qubits in the Kane configuration including A-J-A control gates, auxiliary readout donors and SET readout.

single-gate control paradigm, where the gate operation is limited to the microsecond timescale. In the global control using weak local control and correction paradigm we demonstrate how $(X, Y, Z$, and Hadamard) single qubit operations, and the CNOT gate can be carried out, and provide the actual timescales through numerical simulations. We then discuss readout, scale-up issues and quantum error correction.

\section{SINGLE QUBIT ROTATIONS}

\section{A. Qubit definition}

The architecture of the basic donor electron spin qubit with control gates and a resonant readout mechanism is shown in Fig. 1. Single phosphorus nuclei play a primary role as the localizing centres for donor electron spins which encode quantum information in the canonical fashion as $|0\rangle$ $=|\downarrow\rangle$ and $|1\rangle=|\uparrow\rangle$. To begin with, we analyze the dynamics in the effective spin formalism for which the Hamiltonian for the single qubit system in the absence of a rotating magnetic field is

$$
H_{Q}=\mu_{B} B \sigma_{e}^{z}-g_{n} \mu_{n} B \sigma_{n}^{z}+A\left(V_{A}\right) \boldsymbol{\sigma}_{e} \cdot \boldsymbol{\sigma}_{n},
$$

where $B$ is the strength of the constant magnetic field, $\sigma^{z}$ is the Pauli Z matrix with subscripts $e$ referring to electrons and $n$ referring to the nucleus and $A\left(V_{A}\right)$ is the strength of the hyperfine interaction.

The hyperfine interaction between electron and nucleus is controlled in the usual Stark-shift manner by varying the bias, $V_{A}$, on the $A$ gate in order to deform the electron wave function $\psi\left(r, V_{A}\right)$ around the nucleus thereby changing the hyperfine coupling $A\left(V_{A}\right)$ as $A\left(V_{A}\right) \propto\left|\psi\left(0, V_{A}\right)\right|^{2}$.

It proves beneficial to restrict the Hilbert space of the non-qubit spin: i.e., the nuclear spin space-in our case the lowest energy state corresponding to the nuclear spin up. For the Kane nuclear spin quantum computer the non-qubit electron spins were frozen out by through the large $B=2 \mathrm{~T}$ background field leading to an relative electron spin-up/spindown polarization of $10^{-12}$ at $100 \mathrm{mK}$. Here the field serves a similar purpose, but also relies on the extraordinary long $T_{1}^{(n)} \approx 1 \mathrm{~h}$ of the donor nuclear spin. Since $T_{1}^{(n)}$ is much longer than any other timescale in the system, the nuclear spin once initialized in the up state (lowest energy state) is for all intents and purposes predictably inert. The Kane concept is thus turned inside out.

\section{B. Single qubit Hamiltonian}

We now describe the canonical method of controlling and manipulate electron spins. In Sec. II C we will describe how this method may be improved upon.

By biasing the $A$-gate correctly we are able to select the qubit system (the targeted qubits). In the canonical method, the $A$-gate bias tunes the hyperfine interaction, bringing the qubits into resonance with background if field $B_{\mathrm{ac}}$ and giving us the ability to perform single qubit rotations as required. To gain insight into the canonical control of the electron spin a rf field of frequency $\omega_{\mathrm{ac}}$ we write the single-spin electron Hamiltonian as (assuming frozen nuclear dynamics in the up state):

$$
H_{Q}=\left[\mu_{B} B_{z}+A\left(V_{A}\right)\right] \sigma_{\mathbf{e}}^{\mathbf{z}}+\mu_{B} B_{\mathrm{ac}}\left(\sigma_{\mathbf{e}}^{\mathbf{x}} \sin \omega_{\mathrm{ac}} t+\sigma_{e}^{y} \cos \omega_{\mathrm{ac}} t\right) .
$$

This turns out to be a good assumption for typical parameters expected for the Kane architecture, as we show by numerical simulation including both nuclei and electrons.

For an initial state $|0\rangle$ the well known Rabi solution gives the probability of the electron being found in the state $|1\rangle$ after time $t$ as

$$
P_{1}(t)=\left(\frac{\mu_{B} B_{\mathrm{ac}}}{\Omega}\right)^{2} \sin ^{2}\left(\frac{\Omega t}{\hbar}\right),
$$

where $\Delta \omega=\omega(A)-\omega_{\mathrm{ac}}$ is the detuning between the field quanta and the resonant frequency of the qubit levels $\omega(A)$, controlled by the hyperfine gate $A\left(V_{A}\right) . \quad \Omega(A)^{2}$ $=\left(\mu_{B} B_{\mathrm{ac}}\right)^{2}+\hbar^{2}(\Delta \omega)^{2}$ and the resonant frequency $\omega(A)$ is given to second order by

$$
\omega(A)=2\left(\mu_{B} B+A+\frac{A^{2}}{\mu_{B} B+g_{n} \mu_{n} B}\right) .
$$

In the canonical scheme, being able to perform single qubit rotations is contingent on the ability to shift the electron spin in and out of resonance with the rf field. Calculations show that by applying a voltage to the $A$-gate, one can effectively shift $A .{ }^{20}$ A natural state of operation is to tune the frequency of the rotating magnetic field to the maximally detuned state $\omega_{\mathrm{ac}}=\omega(0)$. In the canonical scheme, when no bias is applied to the $A$ gates, $A=A_{0}$, and each qubit is out of resonance. When a bias voltage is applied, the qubits are forced into resonance with the magnetic field.

In order for the canonical scheme to work, $\Delta \omega$ must be large compared to the full width half maximum (FWHM) of the resonance to achieve fidelities at the $10^{-5}$ level. The FWHM is given by $4 \mu_{B} B_{\text {ac }} / \hbar$. Clearly to locally control spins using this method, we must reduce $B_{\text {ac }}$ at the expense of gate operation time. For an error of $P_{1} \approx 10^{-5}$ for the off resonance qubits (i.e., those not taking part in the operation) one requires $B_{\mathrm{ac}} \approx 10^{-5} \mathrm{~T}$. This leads to a gate operation time of $1.7 \mu$ s for the qubit being addressed. In Table I, this is referred to as the locally controlled electron spin case.

There are several apparent problems with this canonical single-gate scheme with an always on ac field. First, the mi- 
crosecond timescale is slow compared to the natural $Z$ evolution of the electron spins in the $B_{z}=2 \mathrm{~T}$ field. Also, as a result of this fast evolution, one must be able to tune the " $A$ " gate at the frequency of the $Z$ evolution in order to optimize the fidelity of the gates. While this might be possible, we propose an alternative scheme exploiting global control which takes full advantage of the fast timescales.

It is considerably simpler to understand the basic control processes once we transform the single qubit Hamiltonian into a frame rotating with the rf field. We thus make the substitution

$$
|\phi\rangle=\exp \left(\frac{i \omega_{\mathrm{ac}} t}{2} \sigma_{e}^{z}\right)|\psi\rangle
$$

where $|\psi\rangle$ is the wave function in the stationary frame, and $|\phi\rangle$ is the wave function in the frame which rotates at the same frequency as the field $B_{\mathrm{ac}}$. The Hamiltonian in the rotating frame is

$$
\widetilde{H}_{Q}=\hbar \Delta \omega \sigma_{e}^{z}+\mu_{B} B_{\mathrm{ac}} \sigma_{e}^{x} .
$$

The Hamiltonian given in Eq. (6) represents spin precession or rotation around an axis in the $\mathbf{n}=\hbar \Delta \omega \hat{k}+\mu_{B} B_{\mathrm{ac}} \hat{i}$ direction.

In order to take full advantage of the fast timescales in the system we consider an alternative approach for single qubit rotations to the locally controlled case as we anticipate only having limited control over $\omega(A)$. In this proposal we effectively perform single qubit operations by rotating around the $x$ axis (when $\Delta \omega=0$ ) and around an axis which is slightly rotated with respect to this axis described by $\widetilde{H}_{Q}$.

\section{C. $X$ rotations}

In this section we describe the globally controlled qubit operation in the context of an $X$ rotation, by which we mean a rotation around the $\mathrm{x}$ axis. To perform an $X$ rotation, we begin with the resonant magnetic field $B_{\text {ac }}$ tuned to the electron resonance obtained when no voltage is applied to the corresponding $A$-gate $\left(A=A_{0}\right)$, i.e.,

$$
\omega_{\mathrm{ac}}=\omega\left(A_{0}\right) .
$$

In the case when no voltage is applied to an $A$ gate, electrons will undergo a rotation around the $x$-axis $\hat{n}_{0}=\hat{i}$, since $\Delta \omega=0$. They will precess with an angular frequency of $\Omega_{0}=2 \mu_{B} B_{\mathrm{ac}}$. This is the natural frequency of rotation in the system. In the absence of any external influences, every electron precesses at the same rate.

We now consider how to rotate one of the qubits (the target qubit) with respect to the others (the spectator qubits). The speed of rotation of a detuned electron is greater than an electron which is resonant with $B_{\mathrm{ac}}$; that is, $\Omega(A) \geqslant \Omega_{0}$. Therefore, if we detune an electron from the resonance, it will perform a $2 \pi$ rotation in less time than every other qubit requires to do a $2 \pi$ rotation around the $\hat{i}$ axis. In fact every other qubit will undergo a rotation of
TABLE II. Control steps in the single qubit $X$ rotation showing the operations effected on both target and spectator qubits.

\begin{tabular}{cccc}
\hline \hline Step & Target qubit & Spectator qubits & $\begin{array}{c}\text { Time } \\
(\mathrm{ns})\end{array}$ \\
\hline 1 & $I$ & $R_{x}(-\theta)$ & 14.8 \\
2 & $R_{x}(\theta)$ & $R_{x}(\theta)$ & 14.8 \\
Overall & $R_{x}(\theta)$ & $I$ & 29.7 \\
\hline \hline
\end{tabular}

$$
\theta_{x}(A)=2 \pi-\frac{2 \pi}{\Omega(A)} \Omega_{0}=2 \pi-\frac{2 \pi}{\sqrt{\left(\mu_{B} B_{\mathrm{ac}}\right)^{2}+\hbar^{2}(\Delta \omega)^{2}}} \mu_{B} B_{\mathrm{ac}} .
$$

Since $\Delta \omega$ is constrained, the maximum angle which may be rotated in a single step is also constrained. By repeatedly applying this operation and tuning the voltage on the $A$ gate, an $X$ rotation by an arbitrary angle $\theta$ may be constructed. It is convenient to choose $B_{\text {ac }}$ such that any rotation up to $\theta=\pi$ may be performed in a single step. This is possible for typical parameters when $B_{\mathrm{ac}} \leqslant 1.2 \times 10^{-3} \mathrm{~T}$. After this step, the target qubit will not be rotated with respect to its original state, but all the spectator qubits will have undergone a rotation of $R_{x}(-\theta)$.

The second step required is a correction that rotates every qubit, both the target and spectator qubits, by $R_{x}(\theta)$. In this step we bring every qubit in the system into resonance with the magnetic field, and perform an equal $X$ rotation on each qubit. In the first step, each spectator qubit was rotated by $R_{x}(-\theta)$. In the second step everything is rotated by $R_{x}(\theta)$. These angles cancel and therefore no net operation is performed on the spectator qubits. The targeted qubit is effectively not rotated at all by the first step. The second step rotates the targeted qubit by $R_{x}(\theta)$. The targeted qubit therefore has an overall rotation of $R_{x}(\theta)$. The steps required for a full $X$ rotation are shown in Table II.

The overall time required for an $X$ gate [i.e., $\left.R_{x}(\pi)\right]$ is approximately $t_{x}=29.7 \mathrm{~ns}$. Often a correction step can be combined with other correction steps. Not including the correction step (Step 2), the time required for an $X$ gate is around half this value at $t_{x}=14.8 \mathrm{~ns}$. A numerical simulation of this gate was calculated, and a typical evolution is shown in Fig. 3, using the full Hamiltonian including both nuclear and electronic spin.

\section{D. $Y$ rotations}

To achieve a $Y$ rotation, we make use of detuned rotations around the axis:

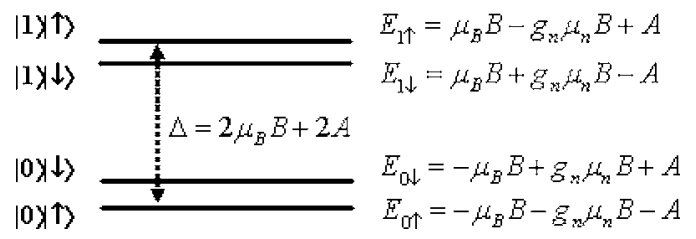

FIG. 2. Energy levels of the donor electron-nucleus system in a magnetic field $B$ and hyperfine coupling $A$. The notation is $\sigma_{e}^{z}=0,1$ (logical qubit states) and $\sigma_{n}^{z}=\uparrow, \downarrow$. 


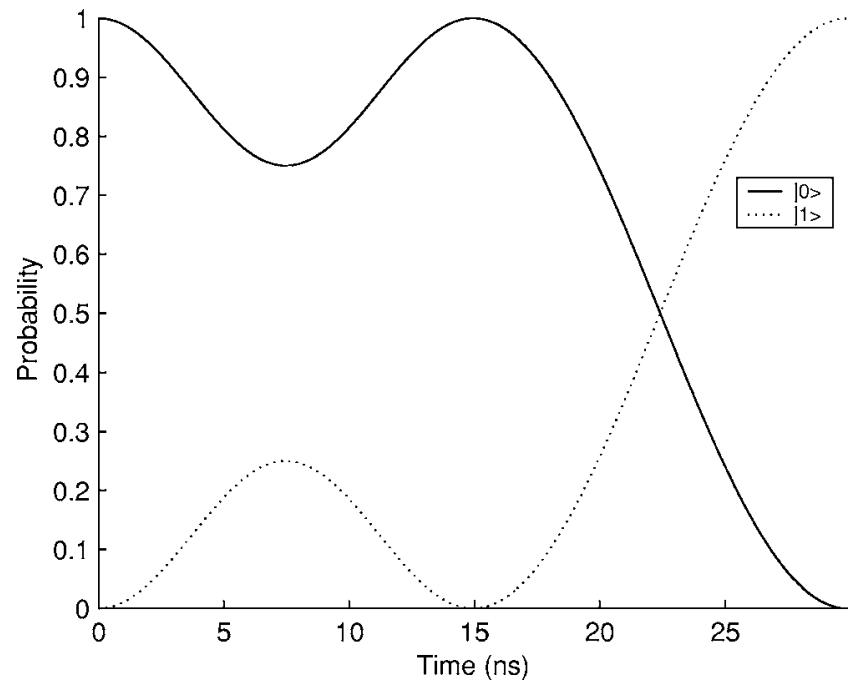

FIG. 3. Typical $X$ gate evolution and timescales for input states as indicated.

$$
\hat{n}=\cos \phi \hat{i}+\sin \phi \hat{k} .
$$

Note, that we can tune the voltage on an $A$ gate and therefore $A\left(V_{A}\right)$ to produce an arbitrary angle $\phi<\phi_{\max }$. The value of $A$ required may be calculated for an arbitrary angle $\phi$, because we know that

$$
\tan \phi=\frac{\Delta \omega}{\mu_{B} B_{\mathrm{ac}}} .
$$

This equation allows us to solve for $\Delta \omega$, and therefore $A\left(V_{A}\right)$. The maximum angle which may be obtained is

$$
\tan \phi_{\max }=\frac{\Delta \omega_{\max }}{\mu_{B} B_{\mathrm{ac}}} .
$$

Now we consider rotations around this axis,

$$
\begin{aligned}
R_{x}(\pi) R_{n}(\pi) R_{x}(\pi) R_{n}(\pi) \\
\quad=(\cos \phi X-\sin \phi Z)(\cos \phi X+\sin \phi Z) \\
\quad=\cos 2 \phi I-i \sin 2 \phi Y=R_{y}(4 \phi) .
\end{aligned}
$$

We may apply $R_{x}(\pi)$ rotations in parallel on every qubit. $R_{n}(\pi)$ rotations may be applied by detuning the target qubit. This technique allows for arbitrary rotations on the target qubit around the $\hat{j}$ axis, up to a rotation of $R_{y}\left(4 \phi_{\max }\right)$. For rotations larger than this angle, one may simply repeat the procedure.

After this operation is complete, a correction step may be required. The target qubit will have undergone a rotation of $R_{y}(4 \phi)$ and all spectators will have been rotated by an angle $R_{x}(\gamma)$ as they are in resonance with the magnetic field during the entire operation. Therefore if the total time of the operation is $t$ then $\gamma=2 \mu_{B} B t$. To correct for this rotation an additional step is required. We rotate the each spectator by $R_{x}(-\gamma)$ and effectively do nothing to the target qubit. This step is identical to the first step when performing an $X$ rotation. Each step in this operation is shown in Table III.
TABLE III. Control steps in the single qubit $Y$ rotation showing the operations effected on both target and spectator qubits.

\begin{tabular}{cccc}
\hline \hline Step & Target qubit & Spectator qubits & $\begin{array}{c}\text { Time } \\
(\mathrm{ns})\end{array}$ \\
\hline 1 & $R_{y}(4 \phi)$ & $R_{x}(\gamma)$ & 50.7 \\
2 & $I$ & $R_{x}(-\gamma)$ & 38.3 \\
overall & $R_{y}(4 \phi)$ & $I$ & 89.0 \\
\hline \hline
\end{tabular}

For typical parameters expected for the Kane architecture a rotation of $R_{y}(\pi)$ will take a total time of $89.0 \mathrm{~ns}$ with the correction step, of which $50.7 \mathrm{~ns}$ is to create the $Y$ gate, and the remaining $38.3 \mathrm{~ns}$ is used to correct the rotation of the target qubit with respect to every other qubit. A typical evolution was numerically simulated and is shown in Fig. 4.

\section{E. Hadamard gate and $Z$ rotations}

Another particularly useful gate in quantum algorithms and quantum error correction is the Hadamard gate. For reference the Hadamard gate is defined for a single qubit as:

$$
H=\frac{1}{\sqrt{2}}\left[\begin{array}{cc}
1 & 1 \\
1 & -1
\end{array}\right]=\frac{1}{\sqrt{2}}(X+Z)=R_{m}(\pi),
$$

where $\hat{m}=1 / \sqrt{2}(\hat{i}+\hat{k})$. We may easily produce this gate by detuning the electron spin from resonance. If we choose $\Delta \omega=\mu_{B} B$ then we will rotate around the axis $\hat{m}$. Similarly to the $X$ and $Y$ gates, the Hadamard gate may require a correction step to cancel any rotation on the spectator qubits. The steps in the Hadamard gate are showing in Table IV. The Hadamard gate takes a total time of $29.7 \mathrm{~ns}$ with the correction applied to the spectators, but a total of only $10.5 \mathrm{~ns}$ without the correction step. The Hadamard gate was simulated numerically, and a typical evolution for this gate is shown in Fig. 5.

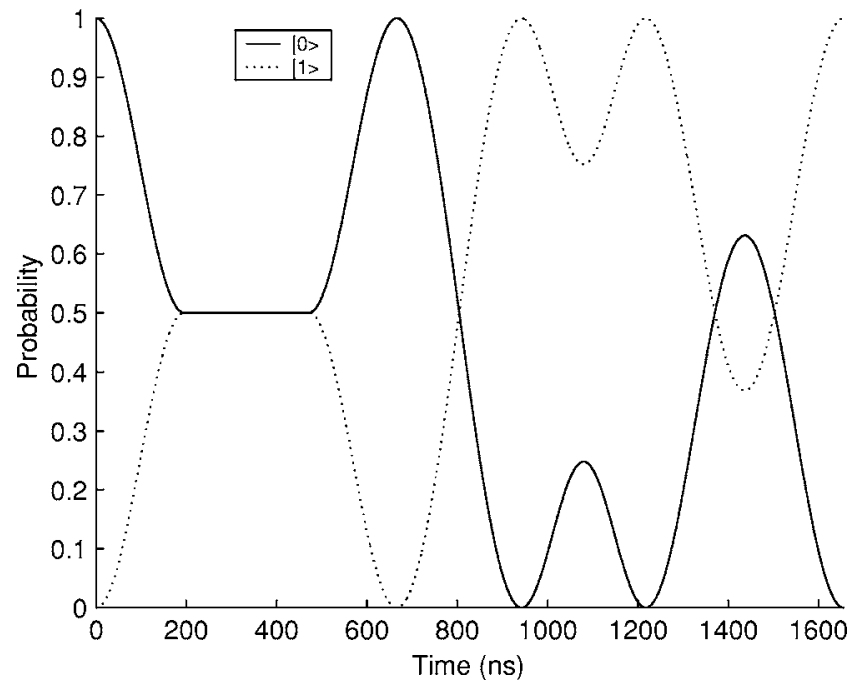

FIG. 4. Typical $Y$ gate evolution and timescales for input states as indiacted. 
TABLE IV. Control steps in the single qubit Hadamard gate showing the operations effected on both target and spectator qubits.

\begin{tabular}{cccc}
\hline \hline Step & Target qubit & Spectator qubits & $\begin{array}{c}\text { Time } \\
(\mathrm{ns})\end{array}$ \\
\hline 1 & $H$ & $R_{x}(\alpha)$ & 10.5 \\
2 & $I$ & $R_{x}(-\alpha)$ & 19.2 \\
Overall & $H$ & $I$ & 29.7 \\
\hline \hline
\end{tabular}

We may perform an arbitrary $Z$ rotation by noting the identity

$$
H R_{x}(\theta) H=R_{z}(\theta)
$$

Therefore we can simply make an arbitrary $Z$ rotation out of existing elements. The steps for this gate are shown in Table V. Only one correction step (Step 4) needs to be applied. The total time required for this gate is $59.4 \mathrm{~ns}$ with the correction step included, and 35.8 ns without the correction step. Again, a typical evolution is shown in Fig. 6.

\section{MULTIPLE QUBIT OPERATIONS}

\section{A. Exchange interaction based CNOT gate}

The exchange interaction and single qubit unitaries may be used to create a CNOT gate. The exchange interaction is proportional to the overlap of the electron wave functions. A simple approximation of the exchange interaction adequate for our purposes, is given by the Herring-Flicker approximation,

$$
J_{\max }(d)=\frac{1.6}{\hbar \epsilon} \frac{e^{2}}{a^{\star}}\left(\frac{d}{a^{\star}}\right)^{5 / 2} \exp \left(-2 \frac{d}{a^{\star}}\right),
$$

where $a^{\star}$ is the effective Bohr radius for the electron, and $d$ is the separation between phosphorus donors. By changing the voltage of the $J$ gate between the phosphorus donors we

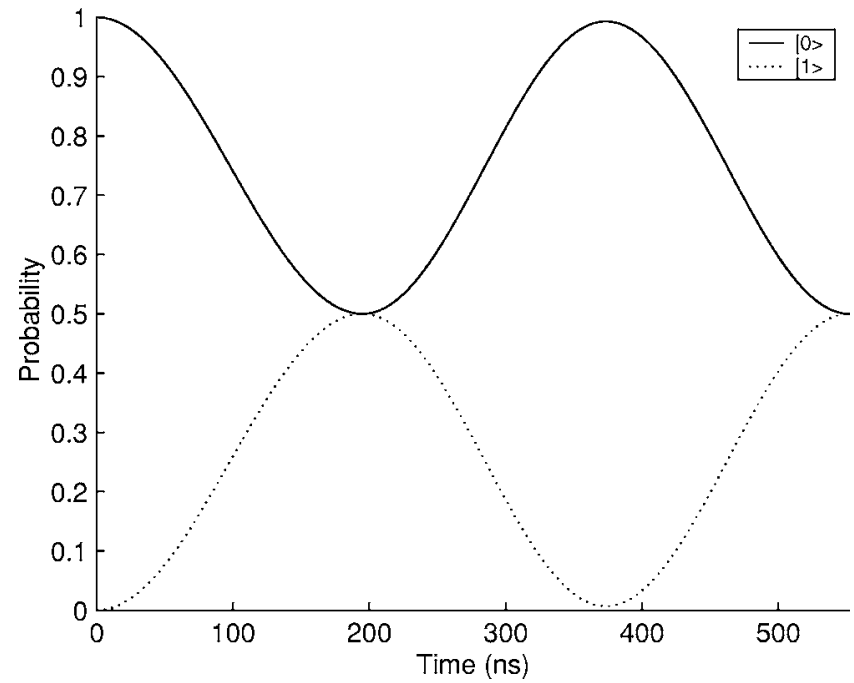

FIG. 5. Typical evolution and timescales of the Hadamard gate for states as indicated.
TABLE V. Control steps in the single qubit $Z$ rotation showing the operations effected on both target and spectator qubits.

\begin{tabular}{cccc}
\hline \hline Step & Target qubit & Spectator qubits & $\begin{array}{c}\text { Time } \\
(\mathrm{ns})\end{array}$ \\
\hline 1 & $H$ & $R_{x}(\alpha)$ & 10.5 \\
2 & $R_{x}(\theta)$ & $R_{x}(\theta)$ & 14.8 \\
3 & $H$ & $R_{x}(\alpha)$ & 10.5 \\
4 & $I$ & $R_{x}(-\theta-2 \alpha)$ & 23.5 \\
Overall & $R_{z}(\theta)$ & $I$ & 59.4 \\
\hline \hline
\end{tabular}

may tune the strength of the exchange interaction $J$ as shown in. ${ }^{21-23}$ Ideally the architecture will be able to tune between $J=0$ and $J=J_{\max }(d)$.

In the rotating frame, the Hamiltonian which includes the exchange interaction is

$$
\tilde{H}_{J}=\mu_{B} B_{\mathrm{ac}}\left(\sigma_{x}^{e_{1}}+\sigma_{x}^{e_{2}}\right)+\Delta \omega_{1} \sigma_{z}^{e_{1}}+\Delta \omega_{2} \sigma_{z}^{e_{2}}+J \boldsymbol{\sigma}^{e_{1}} \cdot \boldsymbol{\sigma}^{e_{2}}
$$

which is particularly simple to manipulate. Note that if $\Delta \omega_{1}=\Delta \omega_{2}$, and in particular if both qubits are tuned to the resonant magnetic field meaning $\Delta \omega_{1}=\Delta \omega_{2}=0$ the identical single qubit rotations commute with the exchange interaction. That is,

$$
\left[\mu_{B} B_{\mathrm{ac}}\left(\sigma_{x}^{e_{1}}+\sigma_{x}^{e_{2}}\right)+\Delta \omega\left(\sigma_{z}^{e_{1}}+\sigma_{z}^{e_{2}}\right), J \boldsymbol{\sigma}_{1} \cdot \boldsymbol{\sigma}_{2}\right]=0
$$

This implies that we may treat the global rotations and the exchange interactions separately.

The controlled sign gate $\Lambda_{1} Z$ may be expressed as

$$
\Lambda_{1} Z=\exp \left(i \pi \frac{I-Z}{2} \otimes \frac{I-Z}{2}\right) .
$$

Using Hadamard gates, the CNOT gate may be expressed as

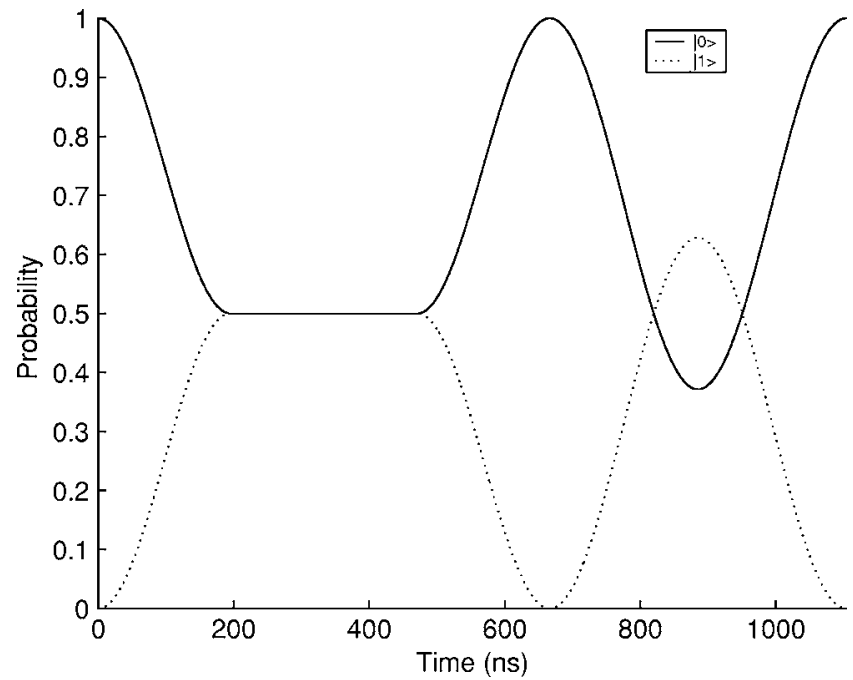

FIG. 6. Typical evolution and timescales of the $Z$ gate for states as indicated. 
TABLE VI. Control steps and times in the exchange based CNOT gate.

\begin{tabular}{ccc}
\hline \hline Step & Operation & Time (ns) \\
\hline 1 & $H \otimes I$ & 29.7 \\
2 & $\exp i \pi / 8 \sigma_{1} \cdot \sigma_{2}$ & 0.01 \\
3 & $X \otimes I$ & 14.8 \\
4 & $\exp i \pi / 8 \sigma_{1} \cdot \sigma_{2}$ & 0.01 \\
5 & $X \otimes I$ & 14.8 \\
6 & $R_{x}(\pi / 2) \otimes R_{x}(\pi / 2)$ & 7.4 \\
7 & $H \otimes I$ & 29.7 \\
8 & Correction & 51.9 \\
Overall & CNOT & 148.4 \\
\hline
\end{tabular}

$$
\begin{aligned}
\Lambda_{1} X= & (I \otimes H) \Lambda_{1} Z(I \otimes H) \\
= & (H \otimes I) \exp \left(i \pi \frac{I-X}{2} \otimes \frac{I-X}{2}\right)(H \otimes I) \\
= & (H \otimes I)\left[R_{x}\left(\frac{\pi}{2}\right) \otimes R_{x}\left(\frac{\pi}{2}\right)\right] \\
& \times \exp \left(i \frac{\pi}{4} X \otimes X\right)(H \otimes I) .
\end{aligned}
$$

Equation (18) is an expression for the CNOT gate which is mostly made up of gates which are straightforward to perform on our architecture, such as the Hadamard, and global $X$ rotations. The only difficult part of this gate is the term $\exp (i \pi / 4 X \otimes X)$ which may be constructed in the following way

$$
\begin{aligned}
\exp \left(i \frac{\pi}{4} X \otimes X\right)= & (X \otimes I) \exp \left(i \frac{\pi}{8} \boldsymbol{\sigma} \cdot \boldsymbol{\sigma}\right) \\
& \times(X \otimes I) \exp \left(i \frac{\pi}{8} \boldsymbol{\sigma} \cdot \boldsymbol{\sigma}\right) .
\end{aligned}
$$

In order to create this interaction correctly, we need to let the qubits interact for a time $t_{J}$ such that $J t_{J}=\pi / 8$.

The largest amount of time in the CNOT gate is in the correction operation. In this step, as in previous gates, we rotate the target qubits with respect to the spectator qubits, and then until the spectator qubits have performed a whole $2 \pi$ rotation. Unfortunately, for the parameters we have chosen, this step turns out to be particularly long. In the absence of this step, the CNOT gate requires only an operation time of $96.5 \mathrm{~ns}$.

The circuit diagram based on this construction (see Fig. 8). The total time required for this gate, based on typical parameters for the Kane architecture is 148.4 ns. A breakdown of the times required for each operation in the gate is shown in Table VI. This gate was simulated numerically, and a typical simulation is shown in Fig. 7. Note that during this gate, corrections need to be performed only when they do not commute with the next gate.

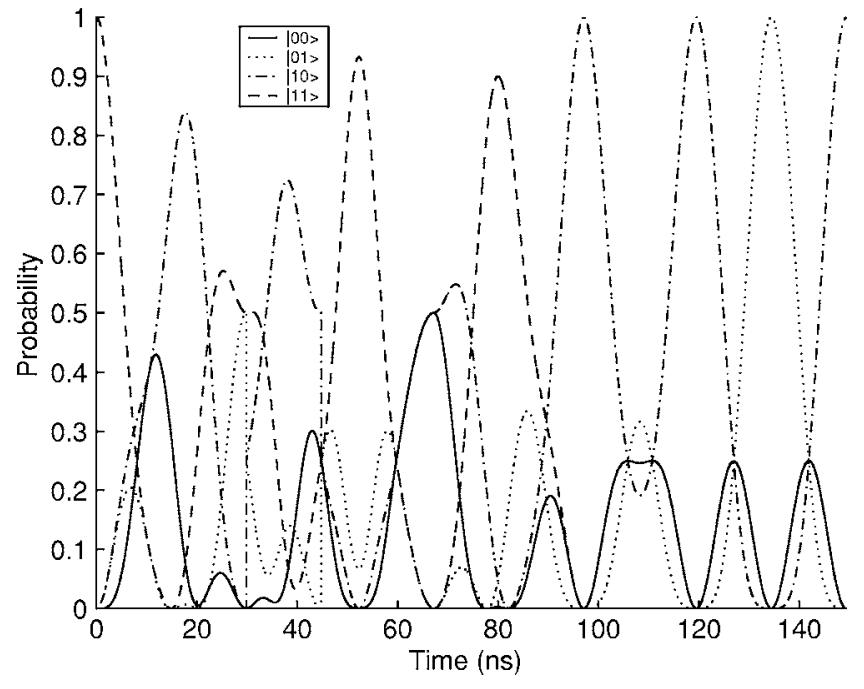

FIG. 7. Evolution and timescales for the CNOT gate for the states as indicated.

\section{B. Swap gate}

The swap gate may be performed particularly easily with the exchange interaction. The swap gate $S$ may be written

$$
S=\exp \left(i \frac{\pi}{4} \boldsymbol{\sigma}_{1} \cdot \boldsymbol{\sigma}_{2}\right) \text {. }
$$

Assuming control of the exchange interaction, this gate may be performed in a single operation with $J t=\pi / 4$. This may then require a correction step. Since this interaction is much larger than the typical frequencies for single qubit rotations, this gate is extremely fast, and to a good approximation does not require a correction step. The speed of this gate also indicates that a three qubit encoding ${ }^{24}$ may be successful.

\section{Dipole-dipole based CNOT gate}

The dipole-dipole interaction couples every pair of electronic spins in the system. The contribution which the dipole-dipole interaction makes to the Hamiltonian is

$$
H_{D}=D\left[\boldsymbol{\sigma}^{e_{1}} \cdot \boldsymbol{\sigma}^{e_{2}}-3\left(\boldsymbol{\sigma}^{e_{1}} \cdot \hat{d}\right) \otimes\left(\boldsymbol{\sigma}^{e_{2}} \cdot \hat{d}\right)\right]
$$

where the strength of the dipole-dipole interaction $D$ is given by

$$
D(d)=\frac{\mu_{0}}{4 \pi} \frac{\mu_{B}^{2}}{d^{3}} .
$$

Whereas the exchange interaction dies off exponentially with distance, as shown in Eq. (14), the dipole-dipole interaction only dies off as $1 / d^{3}$. Therefore at larger separations, the dipole-dipole interaction to dominates.

The direction in which we orientate our qubits w.r.t. the magnetic field $B$ is important. If we align the donors along the $x$ axis $(\hat{i})$ or $y$ axis $(\hat{j}), H_{D}$ does not commute with $\sigma_{z}^{e_{1}}+\sigma_{z}^{e_{2}}$. This implies we may no longer look at our system in a rotating frame. However, if we align our qubits in the $z$ axis $(\hat{k})$ direction then the rotating frame is still valid, and the rotating frame Hamiltonian is 


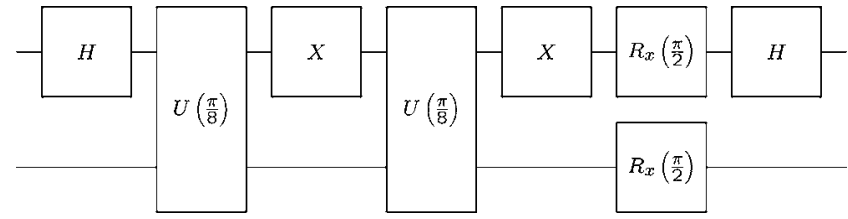

FIG. 8. Circuit diagram for the CNOT gate.

$$
\begin{aligned}
\widetilde{H}_{J D}= & \mu_{B} B_{\mathrm{ac}}\left(\sigma_{x}^{e_{1}}+\sigma_{x}^{e_{2}}\right)+\Delta \omega_{1} \sigma_{z}^{e_{1}}+\Delta \omega_{2} \sigma_{z}^{e_{2}}+J \boldsymbol{\sigma}^{e_{1}} \cdot \boldsymbol{\sigma}^{e_{2}} \\
& +D\left(\boldsymbol{\sigma}^{e_{1}} \cdot \boldsymbol{\sigma}^{e_{2}}-3 \sigma_{z}^{e_{1}} \otimes \sigma_{z}^{e_{2}}\right) .
\end{aligned}
$$

For simplicity we therefore choose to align our axes in the $\hat{k}$ direction.

When the electrons are relatively widely spaced $(d>30 \mathrm{~nm})$ single qubit rotations are much faster than the speed of the interaction $\left(\mu_{B} B_{\mathrm{ac}} \gg D\right)$ and the dipole-dipole interaction dominates the exchange interaction $(D \gg J)$. In order to specify a CNOT gate, we consider the case when the electrons are tuned to the rotating magnetic field, i.e., $\Delta \omega_{1}=\Delta \omega_{2}=0$.

Now

$$
\left[\mu_{B} B_{\mathrm{ac}}\left(\sigma_{x}^{e_{1}}+\sigma_{x}^{e_{2}}\right),(J+D) \boldsymbol{\sigma}^{e_{1}} \cdot \boldsymbol{\sigma}^{e_{2}}\right]=0,
$$

therefore we consider the interaction $(J+D) \boldsymbol{\sigma}^{e_{1}} \cdot \boldsymbol{\sigma}^{e_{2}}$ separately from the single qubit rotations $\mu_{B} B_{\mathrm{ac}}\left(\sigma_{x}^{e_{1}}+\sigma_{x}^{e_{2}}\right)$. Unfortunately the same is not true of the $-3 D \sigma_{z}^{e_{1}} \otimes \sigma_{z}^{e_{2}}$ term in the Hamiltonian where

$$
\left[\sigma_{z}^{e_{1}} \otimes \sigma_{z}^{e_{2}}, \sigma_{x}^{e_{1}}+\sigma_{x}^{e_{2}}\right]=2 i\left(\sigma_{y}^{e_{1}} \otimes \sigma_{z}^{e_{2}}+\sigma_{z}^{e_{1}} \otimes \sigma_{y}^{e_{2}}\right),
$$

for example. Similarly we may calculate higher order commutators. This leads to quite a complicated evolution of the system. Fortunately it is possible to refocus ${ }^{25}$ much of the evolution. However, these higher order terms also anticommute with $\sigma_{x}^{e_{1}} \otimes I$ and therefore we may cancel many of them by conjugation. With this approximation, it is possible to create the CNOT gate using exactly the same pulse sequence as was required when we ignored the dipole-dipole interaction in Sec. III A. The circuit diagram for this circuit is shown in Fig. 8. The interaction is now assumed to be solely due to weak dipole-dipole interaction. In each interaction we must allow the qubits to interact for the comparatively long time of $t_{D}=1 / D \pi / 8$.

At a spacing of $30 \mathrm{~nm}$ we anticipate an extremely long gate time of $4.6 \mathrm{~ms}$. This time is dominated by the time required for the interaction between qubits. A quantum computer based on this scheme has no need for $J$ gates.

\section{CNOT gate with both exchange and dipole-dipole interactions}

In the intermediately spaced regions, neither the dipoledipole nor the exchange interaction dominate. In this case we may use them to complement each other, and create a CNOT gate as described in Secs. III A and III C. In this case the interaction between electrons must be performed for a time of $t_{J D}=1 / J+D \pi / 8$. This leads to a total gate time (for typical parameters at a spacing of $23 \mathrm{~nm}$ ) of $4.0 \mu \mathrm{s}$.
During single qubit rotations it would be beneficial (although not essential) to minimise the exchange interaction. This may be accomplished through the application of voltage to the $J$ gates to isolate the electrons.

\section{PARALLEL GATE OPERATION}

Parallel gates are an essential feature of scalable architectures, and are performed naturally in this scheme. Each of the gates may be performed in parallel. For example $X$ rotations may be performed on two qubits at the same time. This is achieved by simply applying identical control pulses to both qubits. Similarly, identical two-qubit operations may be performed in parallel. For example, a CNOT may be performed between qubits one and two, and between three and four, in parallel.

In addition to applying identical gates to different qubits, many other combinations are possible. Every gate takes a multiple of the period of a spectator qubit (29.7 ns) to perform. After a whole number of periods, the spectators will be in their original orientation. During this time, the correct rotation is applied to the target qubits. The shorter operation being applied in parallel may have to be padded by a number of $2 \pi X$ rotations exactly the same way as the spectators. In this way any two operations which do not act on the same qubits may be applied in parallel. So, for example, an $X$ rotation on qubit 1 , may be performed in parallel with a CNOT on qubits 2 and 3 .

Our scheme takes advantage of two key facts. First, each gate only requires us to change the voltage on the local ' $A$ ' and ' $J$ ' gates. We do not need to modify the magnetic fields, which would affect the operation of other qubits. This means that each operation may be applied independently. Second, each operation takes a whole number of periods of the spectator qubits to perform. Much like a clock in a conventional computer, this greatly simplifies timing issues in performing gates in parallel.

\section{READOUT AND INITIALIZATION}

Readout is a crucial issue to be addressed for donor spin based architectures. We will briefly describe several possible readout schemes here.

Direct single-spin detection is very difficult since a single spin interacts very weakly with its environment and hence the measurement device. In spite of this, magnetic resonance force microscopy (MRFM) has been suggested ${ }^{17,26-28}$ as one of the most promising techniques to achieve such a direct single-spin measurement. Two of the most promising spincantilever modulation protocols to detect a single spin by MRFM are: cyclic adiabatic inversion $(\mathrm{CAI})^{29}$ and oscillating cantilever-driven adiabatic reversal (OSCAR). ${ }^{30}$ The MRFM technique also takes the advantage of the electron spin quantum computer architecture discussed here. The required rf field for the MRFM measurement protocols is also an essential element for the electron spin quantum gate operations. Recently, the MRFM technique has been demonstrated ${ }^{18}$ to detect an individual electron spin. But the required averaging time is still too long to achieve the real- 


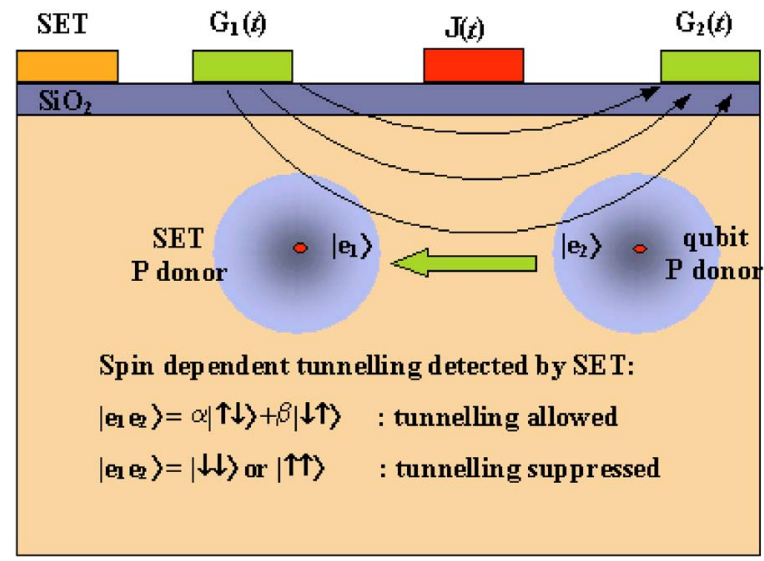

FIG. 9. (Color online) Schematic of the spin-charge transduction process for spin readout using a single electron transistor (SET) as an electrometer.

time readout of the single electron spin quantum state. Given the steady improvement in experimental technique, the MRFM has great potential to serves as an readout device for spin-based qubit systems in the near future.

The spin-charge transduction idea of the original Kane proposal (Fig. 9) called for the adiabatic spin-dependent transfer ${ }^{1}$ of the qubit donor electron to an auxiliary donor leaving a donor ion $D^{+}$and a doubly occupied donor $D^{-}$. The two electron state of this double donor system is conditionally entangled with the original nuclear qubit spin. Detection of the final $D^{+} D^{-}$state by the SET constitutes a measurement of the qubit nuclear spin. A problem with this scheme is the shallow nature of the $D^{-}$state $(1.7 \mathrm{meV})$, which may easily ionize in the electric field required to induce the electron transfer.

The dynamics of the spin dependent transition $D^{0} D^{0}$ $\rightarrow D^{+} D^{-}$was investigated to assess the vulnerability of the adiabatic readout scheme. ${ }^{15} \mathrm{~A}$ comparison to the field strength required for adiabatic transfer, the typical $D^{-}$state dwell-times and SET timescales indicated that adiabatic transfer would at the every least severely test SET measure- ment capability. As a possible alternative to the adiabatic Kane proposal, a resonant-based ${ }^{15}$ scheme has been proposed in which an ac field is applied to the gates $G_{1,2}$ resonant with the transition $D^{0} D^{0} \rightarrow D^{+} D^{-}$. Simulation results indicate a good level of control is achievable for single-qubit addressing in this way using relatively low dc field strengths.

Another alternative ${ }^{19}$ relies on energy resolved readout through the introduction of an ionised donor (the probe) to the usual two donor system for spin readout. Controlling the bias applied to the probe allows resonant charge transfer from either the singlet or triplet state of the combined qubitreference system to the probe. By effecting spin dependent tunneling to the ionized probe, rather than to the reference in the two-donor scheme, we avoid potential problems due to shallow the $D^{-}$state. This can be thought of as using a charge qubit to readout a spin qubit.

\section{CONCLUSION}

We have proposed a scheme for solid state quantum computation based on donor electron spins and global control, using only weak local control. This scheme forms a natural stepping stone and shares similarities with the existing nuclear spin based Kane proposal. We have shown how, even with limited control over the resonant frequencies of the electronic spins and an always on rotating magnetic field, $B_{\text {ac }}$, this system may be used for quantum computation. This scheme outperforms the naive application of the canonical scheme. Indeed, although electron dephasing times are faster than the corresponding nuclear dephasing times, we find that a typical operation time is also correspondingly faster with with $T_{2} / T_{\text {ops }}$ approaching $10^{6}$.

\section{ACKNOWLEDGMENTS}

The authors C.D.H. and H.S.G. would like to thank Gerard Milburn for support. This work was partly supported by the Australian Research Council and by the U.S. National Security Agency (NSA), Advanced Research and Development Activity (ARDA), and the Army Research Office (ARO) under Contract No. DAAD19-01-1-0653.
*Electronic address: hillcd@ physics.uq.edu.au

${ }^{1}$ B. E. Kane, Nature (London) 393, 133 (1998).

${ }^{2}$ R. Vrijen, E. Yablonovitch, K. Wang, H. W. Jiang, A. Baladin, V. Roychowdhury, T. Mor, and D. DiVincenzo, Phys. Rev. A 62, 012306 (2000).

${ }^{3}$ L. C. L. Hollenberg, A. D. Dzurak, C. J. Wellard, A. Hamilton, D. J. Reilly, G. J. Milburn, and R. Clark, Phys. Rev. B 69, 113301 (2004).

${ }^{4}$ A. G. Fowler, C. J. Wellard, and L. C. L. Hollenberg, Phys. Rev. A 67, 012301 (2003).

${ }^{5}$ C. D. Hill and H.-S. Goan, Phys. Rev. A 68, 012321 (2003).

${ }^{6}$ C. D. Hill and H.-S. Goan, Phys. Rev. A 70, 022310 (2004).

${ }^{7}$ A. M. Tyryshkin, S. A. Lyon, A. V. Astashkin, and A. M. Raitsimring, Phys. Rev. B 68, 193207 (2003).

${ }^{8}$ A. J. Skinner, M. E. Davenport, and B. E. Kane, Phys. Rev. Lett.
90, 087901 (2003).

${ }^{9}$ A. A. Larionov, L. E. Fedichkin, and K. A. Valiev, Nanotechnology 12, 536 (2001).

${ }^{10}$ O. Sauret, D. Feinberg, and T. Martin, Phys. Rev. B 69, 035332 (2004).

${ }^{11}$ D. Loss and D. P. DiVincenzo, Phys. Rev. A 57, 120 (1998).

${ }^{12}$ M. Friesen, P. Rugheimer, D. E. Savage, M. G. Lagally, D. W. van der Weide, R. Joynt, and M. A. Eriksson, Phys. Rev. B 67, 121301 (2002).

${ }^{13}$ R. de Sousa, J. D. Delgado, and S. Das Sarma, Phys. Rev. A 70, 052304 (2004).

${ }^{14}$ L. C. L. Hollenberg, C. J. Wellard, and A. D. Greentree, Handbook of Theoretical and Computional Nanotechnology (to be published).

${ }^{15}$ L. C. L. Hollenberg, C. J. Wellard, C. I. Pakes, and A. G. Fowler, 
Phys. Rev. B 69, 233301 (2004).

${ }^{16}$ A. D. Greentree, A. R. Hamilton, and F. Green, Phys. Rev. B 70, 041305 (2004).

${ }^{17}$ T. A. Brun and H.-S. Goan, Phys. Rev. A 68, 032301 (2003).

${ }^{18}$ D. Rugar, R. Budakian, H. J. Mamin, and B. W. Chui, Nature (London) 430, 329 (2004).

${ }^{19}$ A. D. Greentree, A. R. Hamilton, L. C. L. Hollenberg, and R. G. Clark,Phys. Rev. B 71, 113310 (2005).

${ }^{20}$ L. Kettle, H.-S. Goan, S. C. Smith, L. C. L. Hollenberg, C. I. Pakes, and C. Wellard, Phys. Rev. B 68, 075317 (2003).

${ }^{21}$ C. J. Wellard, L. C. L. Hollenberg, L. M. Kettle, and H.-S. Goan, J. Phys.: Condens. Matter 16, 5697 (2004).

${ }^{22}$ C. J. Wellard, L. C. L. Hollenberg, F. Parisoli, L. M. Kettle, H.-S. Goan, J. A. L. McIntosh, and D. N. Jamieson, Phys. Rev. B 68, 195209 (2003).
${ }^{23}$ L. M. Kettle, H.-S. Goan, S. C. Smith, L. C. L. Hollenberg, and C. J. Wellard, J. Phys.: Condens. Matter 16, 1011 (2004).

${ }^{24}$ D. P. DiVincenzo, D. Bacon, J. Kempe, G. Bukard, and K. B. Whaley, Nature (London) 408, 339 (2000).

${ }^{25}$ C. P. Slichter, Principles of Magnetic Resonance (Springer, Berlin, 1990), 3rd ed..

${ }^{26}$ J. A. Sidles, Appl. Phys. Lett. 58, 2854 (1991).

${ }^{27}$ J. A. Sidles, Phys. Rev. Lett. 68, 1124 (1992).

${ }^{28}$ G. P. Berman, F. Borgonovi, H.-S. Goan, S. A. Gurvitz, and V. I. Tsifrinovich, Phys. Rev. B 67, 094425 (2003).

${ }^{29}$ K. Wago, D. Botkin, C. S. Yannoni, and D. Rugar, Phys. Rev. B 57, 1108 (1998).

${ }^{30}$ H. J. Mamin, R. Budkian, B. W. Chui, and D. Rugar, Phys. Rev. Lett. 91, 207604 (2003). 